\title{
Una guía para el desarrollo de un videojuego educativo en educación superior
}

\section{A guide for development of an educational video game in higher education}

DOI: http://dx.doi.org/10.17981/cultedusoc.11.2.2020.05

Recibido: 12 de diciembre de 2019 Aceptado: 13 de marzo de 2020 Publicado: 09 de julio de 2020

Olga Rosalba Rodriguez Jimenez

Universidad Nacional de Colombia. Bogotá, D.C. (Colombia)

orrodriguezj@unal.edu.co

José Ignacio Garcia Pinilla

Universidad Nacional de Colombia. Bogotá, D.C. (Colombia)

jigarciap@unal.edu.co

Para citar este artículo:

Rodríguez, O. y Garcia, J. (2020). Una guía para el desarrollo de un videojuego educativo en educación superior. Cultura, Educación y Sociedad, 11(2). 73-86. DOI: http://dx.doi.org/10.17981/cultedusoc.11.2.2020.05

\section{Resumen}

La investigación tuvo como objetivo sistematizar los componentes estructurales para el desarrollo de un videojuego educativo, desde una aproximación pragmática, a partir de una experiencia con el videojuego (app) sobre métodos cuantitativos desarrollado para estudiantes de Psicología de una universidad pública colombiana. La metodología se basa en la perspectiva de sistematización de experiencias en el ámbito educativo, a la luz de la cual se reconstruyen los principales aspectos de la planeación del videojuego formulados a modo de recomendaciones sobre el proceso. Los resultados inician detallando el propósito del guion, continúan con la presentación de una herramienta de registro denominada Ficha de misión que ayuda a estandarizar el proceso de mapeo de niveles, siguen con algunas consideraciones sobre el equipo de trabajo y finalizan con lecciones aprendidas para su implementación en clase. Se concluye que las pedagogías activas son idóneas para el desarrollo de un videojuego educativo y que las Fichas de misión facilitan la organización de la estructura interna de los niveles, la comunicación entre los profesionales involucrados y el seguimiento de las labores de los desarrolladores del contenido.

Palabras clave: Videojuego; pedagogías activas; educación superior; enseñanza de la Psicología; procesos de desarrollo

\begin{abstract}
The research aimed to systematize the structural elements for development of an educational video game from a pragmatic approach. It based on an experience with the video game (app) on quantitative methods developed for Psychology students at a Colombian public university. Methodologically based on the perspective of systematization of experiences in the educational field, the main aspects of video game planning are critically reconstructed and formulated as recommendations on the process. Results begin by detailing the purpose of script, continue with the presentation of a registration tool called Stage Sheet that helps to standardize level mapping process, continue with some considerations about development team, and close with lessons learned for its class implementation. It is concluded that active pedagogies are ideal for development of an educational video game and Stage Sheets facilitate the organization of the internal structure of levels, the communication between the professionals involved, and the monitoring of content developers' labor.

Keywords: Videogame; active pedagogies; higher edu-
\end{abstract} cation; Psychology teaching; development processes 


\section{INTRODUCCIÓN}

El uso de las tecnologías para la información y la comunicación (TIC) en la educación ha tenido un amplio desarrollo en Latinoamérica como lo señala la Organización de las Naciones Unidas para la Educación, la Ciencia y la Cultura (UNESCO, 2014) a través de modalidades como e-learning, las redes de aprendizaje, los Massive Open Online Course (MOOC) o los seminario en línea, estrategias que además de contribuir a la expansión del conocimiento han contribuido a aumentar la motivación hacia el aprendizaje, la satisfacción con la clase y el desarrollo de la creatividad (Rivero, Chávez, Vásquez y Blumen, 2016). Se ha justificado su uso para el desarrollo de las competencias requeridas por los estudiantes para el ejercicio de su actividad profesional, tales como el trabajo en equipo y el aprendizaje colaborativo.

En relación con el aprendizaje conceptual se ha empleado con éxito para enseñar la conservación de la energía en Ciencias Naturales (Yang, Chien y Liu, 2012), habilidades en el nivel literal de lectura en Lenguaje (Clavijo y Sánchez, 2018), las operaciones básicas (Rojas, 2016), y el concepto de fracción en Matemáticas (Zamora, Lugo y Hurtado, 2018), hasta la tecnología de la prehistoria (Forero y Molano, 2017) y en el diseño y análisis de bases de datos en Ingeniería (Connolly, Stansfield \& McLellan, 2006).

Las TIC han sido fundamentales para el desarrollo de metodologías activas en educación superior, en las cuales la noción de aprendizaje cambia de una perspectiva de transmisión y acumulación de conocimiento a un proceso activo de análisis, creación y resolución de problemas, en el cual adquieren y desarrollan habilidades cognitivas, prácticas y sociales (Jessel, 2013). Por su parte el rol del docente es de facilitador y del estudiante el gestor del propio conocimiento (Salinas, 2004). Desde esta perspectiva, acorde con Holbert y Karadi (2009) el uso de las TIC es coherente con la forma de aprender de los estudiantes quienes son nativos digitales. Camacho (2012) señala que estas metodologías permiten el aprendizaje en profundidad debido a que exige al estudiante "Hacer" y la retroalimentación brindada sobre esta acción le permite alcanzar la comprensión de los conceptos (Higgins, Hartley \& Skelton, 2002).

Una de las propuestas que se ha planteado, dentro de las metodologías activas, se denomina aprendizaje basado en juegos, desarrollados en ambientes informáticos, propuesta que como sistema de aprendizaje implica: 1. Definir los aspectos referidos al aprendiz o modelo de usuario, como los conocimientos o competencias requeridas para iniciar el juego; 2. Plantear la pedagogía que orienta las actividades y problemas a resolver, cómo se ha orientado el diseño de estas actividades y la toma de decisiones en relación con el avance en el juego, la actualización del perfil y los ajustes correspondientes que se deben hacer a partir del avance en el aprendizaje (Gómez-Marín; Gómez-Martín y González-Calero, 2012; Poy-Castro, Mendaña-Cuervo y González, 2015); 3. Contar con un Personaje No Jugador (PNJ) u objeto, el cual cumpla el rol de acompañante pedagógico, que orienta sobre la forma de proceder en el juego y es de particular utilidad en aquellos puntos en los cuales el estudiante no sabe cómo iniciar, continuar o proceder a través de los diferentes niveles del juego (Gómez-Marín et al., 2012).

Se señala también que se debe considerar la relación entre diversión y fantasía, las reglas, las metas, los resultados, la retroalimentación, el conflicto/la competencia; el 
desafío/ la oposición, la interacción, la representación y la narración (Yang et al., 2012; Jessel, 2013). En relación con el diseño se debe considerar la fidelidad, la interactividad y el grado de inmersión, en conjunto con el ambiente, la forma de acceso y los recursos de apoyo que lo acompañan y por supuesto el efecto en el proceso de enseñanza-aprendizaje.

El diseño de este tipo de herramienta pedagógica implica para el docente plantear un conjunto de actividades, ejercicios o retos, definir qué es lo que se va a aprender y en función de ello señalar un criterio de selección de actividades a los que se va a ir enfrentando el estudiante de tal manera que, acorde con las reglas, se logre el objetivo de aprendizaje (Clark, 2004). En relación con la motivación por el aprendizaje, se exige que el juego cuente con un hilo conductor, plantee un final y proponga recompensas parciales y finales (Kirriemuir \& McFarlane, 2004). Estas últimas como parte del sistema de retroalimentación que brinda el juego y que se asocia con la auto-regulación (Ifenthaler, Eseryel \& Ge, 2012). Es importante anotar que los juegos con fines educativos unen objetivos educativos con los de entretenimiento, por lo que están centrados en el estudiante de tal manera que estos tienen un tránsito libre al interior del mismo y en el marco del aprendizaje situado (Jessel, 2013).

En psicología se ha implementado el aprendizaje basado en juegos con distintos propósitos, tales como la aclaración de conceptos (González-Cuevas, 2017) o como en el caso de Martín-Hernández, Agut, Azkue, Gil-Lacruz y Gil-Lacruz (2018) con el aumento de la motivación, el compromiso y el trabajo en equipo, estos autores reportan resultados positivos en el desarrollo y fortalecimiento de procesos cognitivos como la concentración, habilidades para la solución de problemas, la motivación extrínseca, los estados de flujo y el compromiso; aún así debe notarse que los investigadores realizaron un juego simulado en clase y no un videojuego. De manera general, Vlachopoulos y Makri (2017) señalan resultados positivos de la implementación de videojuegos en educación superior tanto a nivel cognitivo, afectivo como conductual.

En el contexto de la enseñanza de la Psicología se conocen pocas experiencias que hagan uso de los videojuegos y reconociendo que aportan al desarrollo de las pedagogías activas, el presente trabajo tiene como objetivo presentar componentes estructurales para el desarrollo de un videojuego educativo, desde una aproximación práctica, a partir de una experiencia con el videojuego (app) sobre Métodos Cuantitativos desarrollado para estudiantes de Psicología de una universidad pública colombiana.

\section{Metodología}

La presentación de la herramienta netamente didáctica que se reconstruye de manera organizada se funda en una investigación cualitativa que adopta la perspectiva de la sistematización de experiencias en el ámbito educativo, uno de cuyos principales objetivos es retroalimentar las orientaciones de programas con los aprendizajes concretos que vienen de diversas experiencias particulares, de manera que las lecciones aprendidas sean semilla de acciones futuras mejor fundamentadas (Jara, 2018).

Las fases sugeridas por Jara (2018) son: 1) Vivir la experiencia; 2) Formular el plan de sistematización; 3) Recuperar el proceso vivido; 4) Realizar reflexiones de fondo y 5) Formular los puntos de llegada. A partir de la recuperación del proceso vivido y las reflexiones de 
fondo, en la sección de resultados se presentan recomendaciones prácticas para educadores interesados en implementar esta estrategia en sus procesos de enseñanza-aprendizaje. Los puntos de llegada que se consideraron pertinentes en la comunicación de la experienciae se refieren a las lecciones aprendidas, que son punto de partida para el desarrollo de nuevos aprendizajes o de futuras experiencias para la creación de videojuegos educativos.

En el marco de la sistematización de experiencias, la credibilidad se aseguró mediante el registro organizado y recurrente de los procesos llevados a cabo en la planeación y posterior desarrollo del videojuego, por lo que se contó con las 25 fichas de misión construidas por un equipo de seis personas, los registros de las reuniones de seguimiento al proceso, los intercambios de información por correo electrónico y las convocatorias realizadas para encontrar los miembros finales del equipo conformado. La dependencia por lo tanto se vinculó a la triangulación de las distintas fuentes de información, durante la reconstrucción histórica del proceso. Finalmente, en la comunicación de los resultados se privilegió el criterio de transferencia, que implica la posibilidad de aplicación de las conclusiones en contextos diferentes (Hernández-Sampieri y Mendoza, 2018).

Aunque desde este enfoque no se visualiza una población o técnicas de validez y confiabilidad, es necesario describir el momento histórico donde tuvo lugar la experiencia. Concretamente, el videojuego Methodi Quantitativae se desarrolló en el Departamento de Psicología de la Universidad Nacional de Colombia, en la sede Bogotá, desde octubre de 2017 hasta abril de 2019, para el curso de Métodos Cuantitativos como parte de las estrategias en pedagogías activas que la docente principal ha venido implementando desde el 2016, pues su interés explícito ha sido mejorar el programa de la asignatura, sus estrategias de enseñanza, la motivación de los estudiantes hacía los contenidos estadísticos, las evaluaciones sumativas y el acompañamiento a los procesos particulares de aprendizaje.

\section{Resultados y Discusión}

A continuación, se presentan los componentes que deben ser considerados para el desarrollo de un videojuego con fines educativos. Inicialmente se detalla el propósito del guion y en quiénes debe recaer su elaboración; continúan con la presentación de la herramienta de registro denominada Ficha de misión, que ayuda a estandarizar el proceso de mapeo de niveles y facilita la comunicación entre los diferentes miembros del equipo de desarrollo; posteriormente se condensan algunas consideraciones sobre el equipo de trabajo, con relación a los mínimos exigibles en el perfil de cada rol atendiendo a las funciones que deben cumplir; finalmente se comentan algunas lecciones aprendidas para la implementación del videojuego en clase.

\section{Consideraciones globales para la elaboración del guion}

La narrativa del videojuego es uno de los elementos centrales ya que crea la atmósfera en la cual ocurrirá la actividad lúdica, además de proporcionar el sentido de: las acciones del jugador, de las conversaciones con los personajes no jugadores (PNJ) y de la cadena de sucesos que ocurran mientras el jugador avanza por el videojuego. En otras palabras, 
es el marco y el lienzo en el cual se plasman los contenidos escogidos, por lo que debe ser atractiva y conseguir que los estudiantes la entiendan, lo que implica que debe ser autocontenida y conectar explícitamente con el contexto de los estudiantes.

El hilo conductor del videojuego debe ser desarrollado por los asesores pedagógicos y ludológicos quienes deben articular en la línea argumental: el objetivo educativo general del videojuego, la cantidad total de actos o episodios de narrativa pura, el tiempo, el lugar, las características del avatar del jugador, el acompañante pedagógico (focalizador de objetivos en un sandbox o videojuego de mundo abierto). Bajo su cargo también recae la identificación y desarrollo de los PNJ, quienes deben tener un sentido para la experiencia global del jugador, así como planificar el prólogo y el epílogo del videojuego, haciéndolo una experiencia sin vacíos.

\section{Composición y contenido de las "Fichas de misión"}

Los escenarios o niveles son las partes principales de un videojuego, en donde se interceptan la narración y la jugabilidad (González, 2016). Cada uno de los niveles que se le proponen al jugador debe mantener un equilibrio entre orden interno, coherencia con toda la línea argumental del videojuego y creatividad. Para lograr este equilibrio, los equipos encargados del diseño de los niveles deben emplear un mismo formato o "Ficha de misión" la cual fue desarrollada con ocasión de la producción del videojuego educativo de Métodos Cuantitativos. La ficha incorpora 17 apartados que permiten de manera organizada desarrollar las ideas sobre el diseño de cada uno de los niveles que se pretende presentar en el videojuego, para el videojuego mencionado, se elaboraron en total 22 fichas de misión para garantizar que se abarcara la totalidad del programa de la asignatura. A continuación, se presenta un modelo de la ficha diligenciada (Tabla 1).

TABLA 1.

Ficha de misión diligenciada para el tema de consideraciones éticas en el proceso de investigación

\begin{tabular}{|c|c|c|c|}
\hline 1. Autor(es) & Fulano Pérez y Mengana López & 17. Área* $N^{\circ}$ & 1 \\
\hline 2. Título de la misión (Tema) & \multicolumn{3}{|c|}{ 1.1 Consideraciones éticas } \\
\hline 3. PNJ involucrado & JUAN PÉREZ & 4. Código** & FM011 \\
\hline 5. Objetivo de aprendizaje & \multicolumn{3}{|c|}{$\begin{array}{l}\text { Se espera que el estudiante aprenda a distinguir situaciones en las } \\
\text { que el código de ética prevalece sobre el propósito de investigación }\end{array}$} \\
\hline $\begin{array}{l}\text { 6. Niveles previos requeridos } \\
\text { (códigos) }\end{array}$ & EH013 & & \\
\hline 7. Ambientación & \multicolumn{3}{|c|}{ Oficina } \\
\hline $\begin{array}{l}\text { 8. Acción del personaje no } \\
\text { jugador en reposo }\end{array}$ & \multicolumn{3}{|c|}{$\begin{array}{l}\text { La profesora se encuentra calificando unos parciales, sentada en el } \\
\text { escritorio con expresión de preocupación. }\end{array}$} \\
\hline \multicolumn{4}{|c|}{ 9. Anotación(es) que el jugador debería conocer previamente (Acompañante pedagógico) } \\
\hline 1 jugador debe encontrar las notas & a profesora en donde se puede lee & & \\
\hline
\end{tabular}




\section{Tarea propuesta por el/la profesor(a) PNJ}

La profesora le solicita al estudiante que revise algunas situaciones dilemáticas propuestas por los estudiantes en los parciales que está calificando y que en cada uno de ellos escoja donde los estudiantes han decidido conforme a la ética psicológica y donde no, clasificando los parciales entre dos carpetas: una con los parciales de decisiones éticas y otra con los parciales de decisiones no éticas.

\section{Medición del avance de la tarea}

La tarea consiste en 10 parciales diferentes, cada uno de los cuales tiene una situación dilemática y una elección propuesta, el jugador debe leer cada parcial y la elección señalada clasificando el parcial por el criterio de elección éticamente adecuada o no adecuada y colocarlos según este criterio en la carpeta correspondiente de entre las dos que tiene a su disposición.

Después de hacer la clasificación de todos los parciales las carpetas brillarán si contienen los parciales correctos, de lo contrario el jugador deberá revisar la clasificación hasta que se distribuyan adecuadamente.

\section{Acción que resuelve la tarea}

La tarea se alcanza si el jugador clasifica adecuadamente cada uno de los parciales entre elecciones adecuadas éticamente e inadecuadas éticamente.

\section{Contenido(s) de la tarea}

El parcial 1 enuncia que (una situación con saliencia del aspecto ético) y la elección que tomó quien escribió el parcial fue (elección entre seguir o para la investigación).

$\cdot \cdot$

..

El parcial 10 enuncia que (una situación con saliencia del aspecto ético) y la elección que tomó quien escribió el parcial fue (elección entre seguir o parar la investigación).

\section{Clave(s) o condición(es) de resolución de la tarea}

Las carpetas brillarán cuando el jugador haya colocado:

1) En la carpeta de elecciones inadecuadas éticamente a los parciales: 1, 3, 5, 7 y 9.

2) En la carpeta de elecciones adecuadas éticamente a los parciales: 2, 4, 6,8, y 10.

\section{Logro(s)}

$\mathrm{Al}$ completar la misión la profesora:

1) Le da una pista para resolver el misterio.

2) Le da unas gafas para aumentar la visión nocturna.

3) Le permite ingresar a las misiones XX112 y YY023.
16. Dificultad de la tarea
1
$2 \quad \mathrm{X} \quad 3$
4
5

*El área se refiere a: 1. Alrededores del edificio; 2. Primer Piso; 3. Segundo Piso.

** El código se construye con las iniciales de los autores y el código de tres dígitos que le corresponde a cada tema del juego en el orden establecido en el Esquema general del contenido, en este caso, como el tema es "1.1" entonces el consecutivo que le corresponde es "011".

Fuente: Autores. 
El apartado 1 incluye a los autores. El apartado 2 se refiere al tema que se pretende enseñar en la misión o nivel, se especifica el aspecto en el que debe centrarse la actividad y permite focalizar las características de la misión a un problema que se ubique en el campo de la temática seleccionada. El apartado 3 señala el PNJ que debe identificar el jugador y con el que debe interactuar para activar el nivel o misión, en la ficha presentada se definió que para la mayoría de los niveles los PNJ fuesen profesores, quienes fueron asociados a sus temas de investigación y las metodologías cuantitativas que se utilizan en sus subcampos disciplinares, pero también pueden ser personajes emblemáticos de la disciplina. La selección de profesores buscó crear un entorno lo más cercano posible a su cotidianidad en la universidad.

En el apartado 4 se etiqueta el nivel completo, en su conjunto, para poder establecer relaciones lógicas entre los diferentes niveles y para facilitar la organización de la programación de los niveles por paquetes lógicos y conectados funcionalmente en el videojuego. En el apartado 5 se especifica el objetivo de aprendizaje del nivel, indicando cuales son los conocimientos sin los cuales el jugador no podría pasar el nivel. El apartado 6 incluye los niveles que deben haber sido jugados antes de poder desarrollar el nivel exitosamente. Este apartado es muy relevante para los temas de mayor complejidad porque ayuda a los programadores a ocultarlos hasta que se pasen exitosamente las misiones que aportan conocimientos necesarios para comprender el tema del nivel o, por otra parte, si son niveles opcionales, de repaso o de profundización de temas, se puede exigir haber realizado exitosamente niveles de menor complejidad.

En el apartado 7 se señala de manera inicial el aspecto gráfico que tendrá la misión y aporta una primera aproximación a lo que se espera ver y lo que dibujantes, diseñadores de las animaciones y de sonido deben empezar a elaborar. El apartado gráfico y audiovisual debe tener un estilo coherente con el tipo de videojuego, por ejemplo, en la Figura 1 se ilustra el aspecto general de la Facultad de Ciencias Humanas, que es el mundo del juego donde se desarrollan las misiones. El apartado 8 contiene una especificación de la actividad que se espera esté realizando en PNJ mientras el jugador no entra en contacto con él, para que no pierda realismo, ni se haga sentir al jugador en un ambiente robotizado.

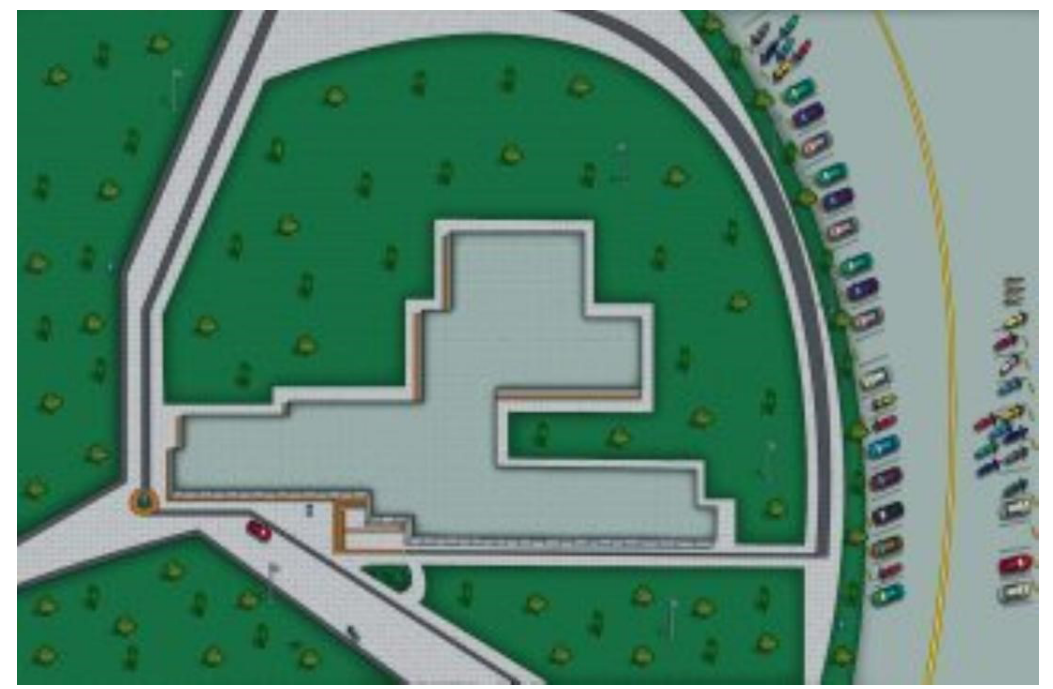

Figura 1. Vista general del mapa completo del videojuego. Fuente: Elaboración propia. 
El apartado 9 contiene información que será vista por el jugador, como pistas, nuevos movimientos, reglas de interacción, normas especiales para el nivel o para lo que resta del videojuego, permiten prever a lo que se va a enfrentar en los próximos niveles. En videojuego educativo es fundamental el acompañante pedagógico, el cual le sugiere al estudiante orientar su atención a conceptos, fórmulas o temas centrales del curso y que dentro del juego le van a permitir avanzar. En el apartado 10 se ubica la instrucción inicial que recibirá el jugador una vez empiece el nivel, debe ser precisa, concisa y clara, se indica en qué consiste la tarea o el problema, para que el jugador pueda pensar la solución más adecuada o darle sentido a los materiales que se presentan durante el nivel.

En el apartado 11 se explica la tarea para los programadores, se especifica el número de casos, fases o elementos que componen el nivel, se indica la retroalimentación al jugador para mantener la motivación hacia el videojuego. En el apartado 12 se señala a los programadores las condiciones lógicas para que el nivel se marque como exitoso, esto es especialmente importante cuando existen niveles en lo que la resolución de todos los casos o elementos no son necesarios, sino que el éxito del nivel se logra al completar un porcentaje o alcanzar una meta preestablecida. En el apartado 13 se relaciona de manera exhaustiva cada uno de los materiales presentes y necesarios para responder a la tarea, se señala el material, el texto, los diálogos entre personajes del videojuego si existen varias fases y todo aquello que se necesite para solucionar el nivel.

En el apartado 14 se hacen explícitas las claves, respuestas o condiciones de resolución exitosa de la tarea propuesta, por cada uno de los casos o elementos a los que debe dar respuesta el jugador. Este apartado informa a los programadores y permite la primera validación del contenido. En el apartado 15 se enumeran las consecuencias concretas para el videojuego una vez el jugador ha solucionado exitosamente el nivel, puede ser a nivel global tal como una pista para solucionar el misterio que enmarca todo el videojuego o algo específico estético o funcional como unas gafas para mejorar la visión nocturna o habilitar otros niveles para que el jugador continúe su trayecto. En el apartado 16 se califica la dificultad del nivel, para equilibrar los conocimientos sobre la temática con la dificultad lógica de la tarea, ya que se espera una progresión en el nivel de dificultad de las tareas, no exclusivamente basado en el conocimiento.

Finalmente, el apartado 17 solo aplica para aquellos videojuegos que utilizan una lógica tipo sandbox, que obliga a definir un mapa para explorar, segmentado en zonas o áreas lógicas, al interior de las cuales se pueden localizar los PNJ que activan el inicio del nivel. Todas tareas deben ser probadas previamente con ayuda de materiales físicos, donde estudiantes que conocen el contenido pueden criticar y ayudar a mejorar aspectos de jugabilidad, coherencia e instrucciones.

\section{Perfiles mínimos para el equipo de trabajo}

Debido a la complejidad del desarrollo de un videojuego se sugiere el siguiente equipo mínimo:

a. Director del proyecto y/o experto en el tema que se pretende enseñar: Por su objetivo, la presencia de un conocedor a profundidad de la temática que da vida al juego es fundamental. Es la persona encargada de supervisar las tareas del equipo y de revisar el contenido temático de todos los niveles para asegurar que las claves sean correctas. 
b. El programador de videojuegos: Este profesional en ingeniería de sistemas es quien debe conocer con todo detalle los pormenores de la programación de un videojuego, Es el responsable de aconsejar sobre el soporte físico, el tipo de lenguaje informático, las consecuencias en términos de costos, desventajas y ventajas de decisiones estéticas, de tamaño del videojuego, de control y seguimiento del avance de los jugadores así como de complejidad lógica de las tareas en los niveles y sus animaciones. También es el responsable de traducir las fichas de misión al lenguaje de programación, de construir la interfaz de control sobre el avance y el acceso al videojuego, así como la comunicación del videojuego con el servidor web que almacene todos los datos. Finalmente, es la persona encargada de realizar el soporte técnico en las plataformas y de entregar todo el material informático al director, para que este pueda mantener el control del videojuego en todo momento.

c. El asesor pedagógico: Este profesional de las ciencias de la educación es el responsable de supervisar que la estructura del videojuego responda a los objetivos educativos predefinidos, debe señalar la organización cognitivamente razonable de los niveles, la estructura lógica del videojuego y de los niveles en su interior para promover el aprendizaje autónomo del grupo etario al cual se enfoca, la priorización de aspectos al interior de los temas seleccionados y el tipo de lenguaje más recomendable a utilizar en los diálogos. También debe coordinar la elaboración de los niveles y retroalimentar a nivel pedagógico cada una de las tareas propuestas y apoyar la comunicación con el programador para que la traducción de la ficha de nivel al videojuego mantenga las propiedades educativas que le son esenciales. Finalmente, es la persona más idónea para resumir el proceso analíticamente y para equilibrar el peso lúdico que suele emerger con fuerza durante el desarrollo del proyecto.

d. El asesor ludológico: Este profesional de los estudios sobre videojuegos es el responsable de hacer que el videojuego no pierda su carácter lúdico al priorizar el interés de aprendizaje. Debe señalar la estética general del videojuego, el género lúdico más apropiado, la línea narrativa más coherente, así como los detalles que motivan a diferentes tipos de jugadores a avanzar en el videojuego. Durante el proceso, es crucial en la coordinación de los desarrolladores de contenido y su retroalimentación ludológica, adicionalmente, debe ser un aliado del programador para que la estructura general del videojuego y de cada uno de los niveles no pierda su carácter lúdico. Finalmente, es la persona más idónea para acompañar el proceso estético y equilibrar el peso educativo del videojuego.

e. El ilustrador: Profesional de las artes digitales responsable de establecer la estética gráfica del videojuego, a su cargo corre el dibujo de los personajes, los escenarios, los materiales de las tareas llegando hasta los botones de la pantalla de inicio, el tipo de fuente de los textos y las opciones del HUD, esto último referido a mapas, puntos, indicaciones permanentes o temporales en la pantalla. Debe estar familiarizado con el tipo de arte que se espera desarrollar ya sea 2D ó 3D. Su responsabilidad es muy elevada ya que el aspecto general del videojuego se infiere desde la primera imagen que ve el jugador, como la pantalla de inicio que se presenta en la Figura 2. 


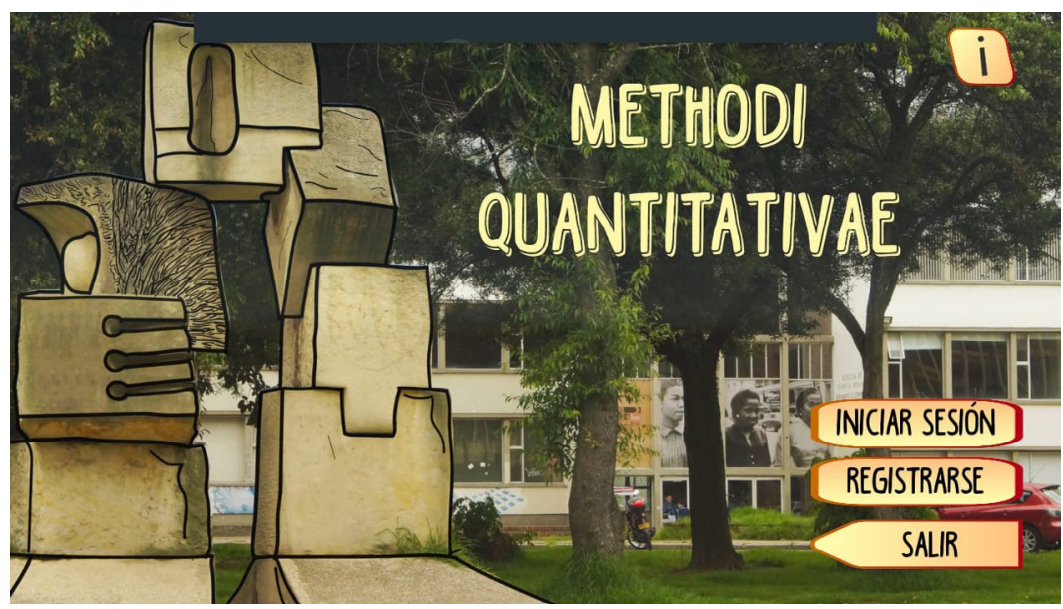

Figura 2. Pantalla de inicio del videojuego.

Fuente: Elaboración propia.

f. El mezclador: este profesional de la ingeniería acústica es el responsable de desarrollar, grabar y supervisar todos los sonidos que aparezcan en el videojuego, desde la música en la pantalla de inicio, las cinemáticas, la música de fondo constante, hasta los diálogos de los PNJ, los sonidos ambientales, de movimiento y del HUD o la configuración. Debe trabajar en coordinación con el ilustrador dado que la música debe corresponderse culturalmente con las expectativas gráficas y el sentido del videojuego para mantener la misma estética.

g. Los desarrolladores de contenido o mapeadores: son profesionales o estudiantes avanzados responsables de crear los niveles, deben tener experiencia como jugadores, aprendices y pedagogos. Su labor se apoya en las fichas de misión para unificar el lenguaje. Se recomienda el trabajo en parejas para mantener un control por pares de las ideas, conocimientos y desarrollos que realicen.

Otras personas que suelen ser esenciales en el desarrollo de un videojuego educativo son los patrocinadores, quienes aportan el dinero y los vínculos institucionales para implementar la herramienta; los actores de voz, profesionales que actúan los diálogos de los personajes del videojuego; los modelos, personas reales que sirven de inspiración para construir los PNJ cercanos al contexto de la institución educativa, los tester o ensayadores, estudiantes que prueban las tareas antes de que apruebe la ficha de misión y el videojuego antes de que se apruebe completamente, buscando fallas técnicas, lógicas y pedagógicas activamente en los dos casos y los escritores, quienes sugieren la línea narrativa más pertinente para los objetivos y características del videojuego.

\section{Articulación pedagógica dentro de la clase}

Se debe incluir en el proceso de enseñanza-aprendizaje del curso que, por su naturaleza lúdica, se propone como estrategia de evaluación formativa, con lo cual se puede habilitar un grupo de niveles al finalizar cada unidad temática del curso, mediante el seguimiento por parte de monitores o compañeros, en consideración a la importancia que tiene la instrucción entre pares (Pinagorte, 2014) y la efectividad de su implementación en ambientes universitarios (Escudero, 2014). El enfoque para el ámbito universitario debe atender 
especialmente al tipo de población adolescente y adulta joven que hace parte de estos escenarios, minimizando las relaciones asimétricas y buscando que encuentren un sentido práctico para cada uno de los aprendizajes que se proponen (Knowles, 1980), de manera que se vincule el videojuego como una herramienta en la que también se simulen estos contextos reales donde el conocimiento en métodos cuantitativos tiene un sentido pragmático.

En términos de sostenibilidad se requiere del soporte permanente por parte de los ingenieros de sistemas, incluso cuando existe una interfaz que automatiza el registro y seguimiento de los estudiantes por parte del docente, ya que se requiere la corrección de incompatibilidades con sistemas y plataformas, como también por las actualizaciones que el docente pueda realizar al contenido del curso. Asimismo, el tiempo dedicado a las actividades relacionadas directamente con el videojuego en la clase se considera de 20 minutos semanales de tal manera que al concluir la unidad temática que dura aproximadamente un mes se hayan finalizado los niveles de juego respectivos, lo que permite un avance relativamente sincronizado del videojuego con las clases.

\section{CONCLUSiOnes}

El desarrollo de un videojuego educativo requiere esfuerzos humanos, materiales, económicos y creativos similares a los necesarios para de un videojuego comercial. Sin embargo, se requiere de una configuración que recoja las necesidades educativas y cognitivas de estudiantes de nivel universitario, lo cual implica que los principios de aprendizaje deben asentarse en la andragogía, en el aprendizaje para adultos. De manera más específica el enfoque de las pedagogías activas se convierte en el eje idóneo para la formulación de la didáctica y la lógica general del proceso educativo y de enseñanza-aprendizaje que tiene lugar en interfaces digitales como los videojuegos.

Los videojuegos educativos son herramientas con un alto potencial como objeto de aprendizaje que requiere de mayores desarrollos conceptuales y prácticos en el ámbito universitario. Las pedagogías activas, que exigen seguimiento y retroalimentación continua, se pueden hacer efectivas con mayor facilidad con las posibilidades que se ofrecen en los videojuegos: seguimiento de la actividad del jugador, cálculo de puntuaciones según el nivel avanzado, número de intentos necesarios para lograr el éxito en las tareas, tipo de errores más frecuentes entre los jugadores; con los cuales el profesor puede orientar su clase magistral o el acompañamiento in vivo necesario para subsanar los vacíos conceptuales que se presentan en el grupo.

En términos de desarrollo, la utilización de la "Fichas de misión" es una alternativa eficaz de comunicación interdisciplinaria, ya que facilita la articulación de las facetas de jugabilidad y narrativa que se encuentran de manera simultánea en la elaboración de los escenarios, dando un sentido coherente a los elementos presentes dentro de los niveles con la estructura general del videojuego, sin disminuir las posibilidades creativas. Así mismo, funciona como una forma estándar de hacer el seguimiento al equipo mapeador y de reconstruir el escenario para los ingenieros de desarrollo. Adicionalmente, la decisión de desarrollar una app como forma de presentación del videojuego, funcional también mediante emuladores en otros dispositivos, responde a las tendencias sociales y educativas de Latinoamérica, lo que facilita su acceso y utilización en el nivel universitario. 


\section{Agradecimientos}

Este artículo hace parte del proyecto financiado por la Convocatoria de Investigación sobre Innovación Pedagógica de la Universidad Nacional de Colombia (Sede Bogotá) en 2016.

\section{REFERENCIAS}

Camacho, M. (2012). El uso de mandos interactivos: una innovación docente para aumentar la motivación y mejorar el aprendizaje del alumnado universitario. Revista Teoría de la Educación: Educación y Cultura en la Sociedad de la Información, 13(1), 412436. Recuperado de http://revistas.usal.es/index.php/eks/article/view/8818/9022

Clark, C. (April, 2004). The Principles of Game Based Learning. NETC / LSC Conference, Crystal City, VA, EUA.

Clavijo, S. y Sánchez, L. (2018). La competencia lectora en el nivel literal a través de una estrategia pedagógica apoyada por un objeto virtual de aprendizaje, en los estudiantes del curso 302, de la jornada de la mañana del colegio distrital Rodolfo Llinás. [Tesis de Maestría]. Universidad Libre, Bogotá, D.C., Colombia. Disponible en http:// hdl.handle.net/10901/11613

Connolly, T., Stansfield, M. \& McLellan, E. (2006). Using an Online Games-Based Learning Approach to Teach Database Design Concepts. The Electronic Journal of e-Learning, 4(1), 103-110. Recuperado de http://www.ejel.org/issue/download.html?idArticle=19

Escudero, R. (2014). Impacto del método "Instrucción por pares" con el apoyo de "clickers" en el aprendizaje de Matemáticas Básicas. Revista Internacional de Tecnología, Conocimiento y Sociedad, 3(1), 57-68. https://doi.org/10.37467/gka-revtechno.v3.1180

Forero, A. y Molano, X. (2017). Tecnología de la prehistoria en el altiplano cundiboyacense, propuesta de un Objeto Virtual de Aprendizaje para Ciclo 2 (Chía y Zuhe). [Trabajo de grado]. Universidad Pedagógica Nacional, Bogotá, D.C., Colombia. Recuperado de http://hdl.handle.net/20.500.12209/9772

Gómez-Marín, M., Gómez-Martín, P. y González-Calero, P. (2012). Aprendizaje basado en juegos. Revista ICONO14 Revista Científica de Comunicación y Tecnologías Emergentes, 2(2), 1-13. https://doi.org/10.7195/ri14.v2i2.436

González, D. (2016). Diseño de videojuegos. Da forma a tus sueños. Bogotá, D.C.: Ra-MaEdiciones de la U.

González-Cuevas, G. (october, 2017). Common misconceptions about psychopharmacology in undergraduate psychology and pharmacy students. 8th International Conference on Education and Educational Psychology, Future Academy, Porto, Portugal. http:// dx.doi.org/10.15405/epsbs.2017.10.75

Hernández-Sampieri, R. y Mendoza, C. (2018). Metodología de la investigación. Las rutas cualitativa, cuantitativa y mixta. México, D.F.: McGraw Hill Education.

Higgins, R., Hartley, P. \& Skelton, A. (2002). The Conscientious Consumer: Reconsidering the role of assessment feedback in student learning. Studies in Higher Education, 27(1), 53-64. http://dx.doi.org/10.1080/03075070120099368 
Holbert, K. \& Karady, G. (february, 2009). Strategies, challenges and prospects for active learning in the computer-based classroom. IEEE Transactions on Education, 52(1), 33-38. http://dx.doi.org/10.1109/TE.2008.917188

Ifenthaler, D., Eseryel, D. \& Ge, X. (Eds.) (2012). Assessment in game-based learning: Foundations, innovations, and perspectives. New York: Springer.

Jara, O. (2018). La sistematización de experiencias: práctica y teoría para otros mundos posibles. Bogotá, D.C.: Centro Internacional de Educación y Desarrollo HumanoCINDE.

Jessel, J. (2013). Procesos sociales, culturales y cognitivos y nuevas tecnologías en educación. En, O. Miglino, M. Nigrelli y L. Sica (Eds.) Videojuegos de rol, simulaciones por ordenador, robots y realidad aumentada como nuevas tecnologías para el aprendizaje: guía para profesores, educadores y formadores. Castelló de la Plana: Publicacions de la Universitat Jaume I.

Kirriemuir, J. \& McFarlane, A. (2004). Literature review in games and learning. Bristol: Nestafuturelab.

Knowles, M. (1980). The modern practice of adult education: From pedagogy to andragogy. New York: Cambridge.

Martín-Hernández, P., Agut, S., Azkue, J., Gil-Lacruz, M. \& Gil-Lacruz, A. (july, 2018). Enhancing motivation, engagement and team building among psychology undergraduates through game base learning. En, L. Gómez, A. López, I. Candel (ed. lit.), Proceedings of EDULEARN18 Conference, Palma, Mallorca, España. http://dx.doi. org/10.21125/edulearn.2018.2336

Pinagorte, K. (2014). Instrucción entre pares, un método sencillo pero efectivo para enseñar. Revista FENopina, 58(1), 56-59. Disponible en http://www.revistas.espol.edu.ec/ index.php/fenopina/article/view/49

Poy-Castro, R., Mendaña-Cuervo, C. y González, B. (2015). Diseño y evaluación de un juego serio para la formación de estudiantes universitarios en habilidades de trabajo en equipo. RISTI, 3(Especial), 71-83. http://dx.doi.org/10.17013/risti.e3.71-83

Rivero, C., Chávez, A., Vásquez, A. y Blumen, S. (2016). Las TIC en la formación universitaria. Logros y desafíos para la formación en psicología y educación. Psicología, 34(1), 185-199. http://dx.doi.org/10.18800/psico.201601.007

Rojas, J. (2016). Diseño de un Objeto Virtual de Aprendizaje para la resolución de problemas matemáticos con las operaciones básicas adición, sustracción, multiplicación y división. [Tesis de especialización]. Universidad Pedagógica Nacional, Bogotá, D.C., Colombia. Recuperado de http://hdl.handle.net/20.500.12209/9573

Salinas, J. (2004). Innovación docente y uso de las TIC en la enseñanza universitaria. Revista universidad y sociedad del conocimiento, 1(1), 1-15. http://dx.doi.org/10.7238/ rusc.v1i1.228

Vlachopoulos, D. y Makri, A. (2017). The effect of games and simulations on higher education: a systematic literature review. Technology in Higher Education, (14), 2-33. https://doi.org/10.1186/s41239-017-0062-1

UNESCO. (2014). Políticas TIC en los Sistemas Educativos de América Latina. Informe sobre Tendencias Sociales y Educativas en América Latina. París: UNESCO. Recuperado de https://unesdoc.unesco.org/ark:/48223/pf0000230080 
Yang, J., Chien, K. \& Liu, T. (2012). A digital game-based learning system for energy education an energy conservation pet. The turkish online Journal of educational technology, 11(2), 27-37. Recuperado de http://www.tojet.net/articles/v11i2/1123.pdf

Zamora, J., Lugo, L. y Hurtado, S. (2018). Diseño de un objeto virtual de aprendizaje (OVA) e implementación de Scratch para favorecer el aprendizaje significativo del concepto de fracción, en los estudiantes de grado $5^{\circ}$ de la Institución Educativa Presbíterio Horacio Gómez Gallo, sede Santa Cecilia. [Trabajo de grado]. Universidad Santiago de Cali, Santiago de Cali, Colombia.

Olga Rosalba Rodriguez Jimenez es Psicóloga de la Universidad Nacional (Colombia) con maestría en Educación de La universidad Javeriana (Colombia). Magister en ciencias del comportamiento y salud, y doctora en Desarrollo psicológico, aprendizaje y educación de la Universidad Autónoma de Madrid (España). Docente, investigadora y consultora en Psicometría, métodos cuantitativos en psicología y educación. Además investiga y diseña procesos de evaluación y mejora escolar, con énfasis en la construcción y análisis estadístico de instrumentos de medición y evaluación del aprendizaje, como en la coordinación de procesos de evaluación masiva con propósitos laborales y, apoya en el diseño, implementación y evaluación de procesos en instituciones educativas. https://orcid. org/0000-0003-4099-5535

José Ignacio Garcia Pinilla es Psicólogo de la Universidad Nacional (Colombia). Aspirante al título profesional en Sociología y maestrante en Psicología, con énfasis en evaluación y mejora de procesos educativos en la misma institución. Ha desarrollado investigaciones en el ámbito educativo y pedagógico aplicando métodos cuantitativos, cualitativos y mixtos de investigación. Sus intereses académicos incluyen las pedagogías activas y la educación para adultos (andragogía). https://orcid.org/0000-0002-7018-3776 